\title{
Preface: Metastable alloys
}

\author{
Jun-wei Qiao'
}

Published online: 5 March 2018

(C) China Iron and Steel Research Institute Group 2018

With the increase in the cooling rate during solidification, ultrafine grained alloys (micrometer and nanometer scale) and even amorphous alloys would form as a result. Thermodynamically, they are not stable and subjected to effective heat treatment. These alloys could experience coarsening (grain growth), crystallization, phase transformation, etc. Besides, under magnetic, electric, and mechanical fields, the alloys including thermally unstable alloys may also have structure and property transition. The above-mentioned alloys can be called metastable alloys.

In this issue, five papers dealing with metastable alloys are published. Zhang et al. studied the giant magnetoimpedance effect (GMI effect) of four Co-based fibers connected in the frequency range of $0.1-10 \mathrm{MHz}$ and the influence of distance between adjacent fibers on the distribution of circumferential magnetic field in every fiber surface. Shen et al. fabricated melt-extracted $\mathrm{Gd}_{50} \mathrm{Al}_{30} \mathrm{Co}_{20}$ amorphous wires with diameter of $\sim 30 \mu \mathrm{m}$ with excellent magnetocaloric properties and average fracture strength of $\sim 969.5 \mathrm{MPa}$, best fitted by Weibull probability distribution. The $\mathrm{Fe}_{78} \mathrm{Si}_{8} \mathrm{~B}_{14}$ and $\mathrm{Fe}_{78} \mathrm{P}_{8} \mathrm{~B}_{14}$ ribbons with different wheel speeds were prepared by melt-spinning, and their responses on $\mathrm{He}^{+}$ions irradiation were investigated by Wei et al. The size and the fraction of precipitate phases in fully amorphous matrix play a dominated role in resisting the ion irradiation effects. Luo et al. investigated the strong textured commercially pure titanium alloy TA2 plates with different initial textures rolled at the cryogenic and ambient temperatures to the reduction of $4 \%$ and then post-annealed at the temperature of $50{ }^{\circ} \mathrm{C}$ for $12 \mathrm{~h}$. The mechanical enhancement of the sheets is attributed to the massive twins and low-density dislocations in the microstructure. Bai et al. studied the wear behavior of Ti-based metallic glass matrix composites and found that the wear rate presented a negative linear correlation with increasing normal loads under dry conditions and simulated seawater.

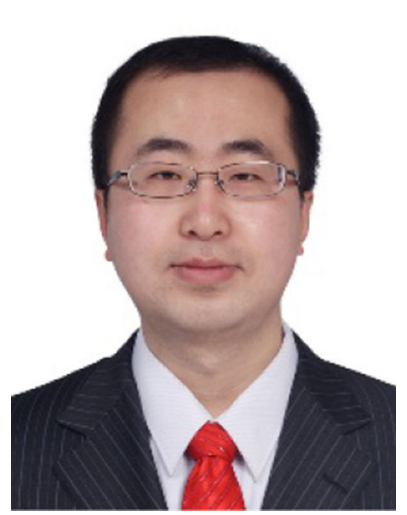

Jun-wei Qiao, Professor is with Taiyuan University of Technology. Dr. Qiao, as the group leader in Lab of Metastable Alloys, is mainly working on mechanical behavior of amorphous alloys and high-entropy alloys. Up to now, Dr. Qiao has published more than 120 papers in Materials Science and Engineering R: Reports, Acta Materialia, Scientific Reports, etc., and edited two book chapters.
Jun-wei Qiao

qiaojunwei@gmail.com

1 Research Center for High-entropy Alloys, College of Materials Science and Engineering, Taiyuan University of Technology, Taiyuan 030024, Shanxi, China 